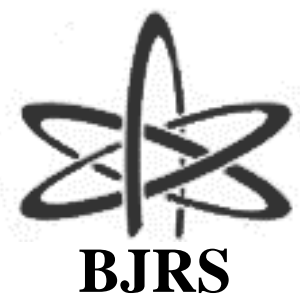

BJRS
BRAZILIAN JOURNAL

$\mathrm{OF}$

RADIATION SCIENCES

09-01 (2021) 01-15

\title{
X-RAY SPECTROMETRY FROM A MICROCT
} SYSTEM

\author{
Hugo C. Romberg Jr ${ }^{1}$, Caio M. S. F. F. Santos ${ }^{1}$, Tâmara P. Teixeira ${ }^{1}$, Olga M. O. Araújo ${ }^{1}$, \\ Davi F. Oliveira ${ }^{1}$ and Ricardo T. Lopes ${ }^{1}$ \\ ${ }^{1}$ Nuclear Instrumentation Laboratory, \\ Federal University of Rio de Janeiro \\ P.O Box 68509 \\ 21941-972 Rio de Janeiro, RJ, Brazil \\ hugoromberg@poli.ufrj.br
}

\begin{abstract}
$\mathrm{X}$-rays are a form of radiation of fundamental importance in a wide variety of applications, and among them is the microcomputed tomography (microCT). MicroCT is a nondestructive technique used in many industrial and research areas such as geology, archeology and biomedicine. In this work the spectra of the v|tome|x m tomographer were acquired and analyzed in order to better understand the energy spectra of a microCT X-ray tube. A semiconductor detector, model XR-100CdTe, was used for data acquisition. Energy spectra were acquired using different filtering and tube voltage configurations. Filtering systems were comprised of aluminum and copper metallic filters. Unfiltered spectra acquisition was performed for tube potential ranging within 20-80 $\mathrm{kV}$, and filtered spectra at tube voltage of $40-120 \mathrm{kV}$, with a $10 \mathrm{kV}$ step. Results also include average energy values of unfiltered and filtered beams for tube potential of $40-120 \mathrm{kV}$, as well as the attenuation value of $60 \mathrm{kV}$ and $70 \mathrm{kV}$ related spectra regarding each of the filtering systems, which altogether enabled the evaluation of how the use of filters impact $X$-ray tube energy spectra.
\end{abstract}

Keywords: $x$-ray, spectrometry, microCT 


\section{INTRODUCTION}

The capacity of X-rays to interact with matter makes usage of this type of radiation applicable in various situations. This capacity varies according to the density of the substance to be observed. In addition, the penetrating power of X-rays also depends on the energy spectrum of the beam. Amongst its many applications are medical and industrial radiography. Computer tomography (CT) is widely used for medical purposes, and microcomputed tomography (microCT) are both of great importance to various fields, among them, but not limited to, dentistry, archeology and biomedicine [1].

In many situations, in both $\mathrm{CT}$ and microCT, filters are added to the system as a way of removing low energy photons to obtain better image results and/ or to reduce patient exposure to radiation. The detector in a microCT system has a limit of events it can process per time unit, limit that when reached causes saturation of said system. In order to avoid detector saturation under certain circumstances, metallic filters can be used to eliminate low energy photons from the energy beam. The use of metallic filters may also aid in the correction of what is known as the beam hardening artifact, which compromises the quality of the microtomography image [2].

This paper aims for a better understanding of the spectrum generated by an X-ray tube used in a microCT system and the way the spectrum is affected by the use of filters. A tungsten target, microfocused tube with maximum tension of $300 \mathrm{kV}$ was used in this experiment.

The spectra were acquired in various filter and beam configurations, with different values of maximum energy. Among the tensions used are 60 and $70 \mathrm{kV}$, which are used in various works [38]. In [3] a $1 \mathrm{~mm}$ thick aluminum filter was used for dentistry tomography, while in [4] no filter was used in the tomography of rabbits. Reaching for a better result in image quality, the use or not of filters must be evaluated in each circumstance. These spectrums were obtained by a cadmium telluride (CdTe) semiconductor type detector, as in previous works before [9, 10] and the attenuation values corresponding to each filtering system were calculated for certain energy intervals, as well as the average energy associated to each analyzed X-ray beam. 


\section{MATERIALS AND METHODS}

The choice of a cadmium telluride (CdTe) semiconductor detector is justified by its high stopping power, a determining factor for the realization of this study due to its X-ray attenuation coefficient [11]. The term stopping power refers to the average loss of energy per unit length in a specific environment and it depends on the type of material and incident radiation [12]. The XR100CdTe system, manufactured by Amptek, consists on a high performance X-ray detector, a preamplifier and a cadmium telluride cooler measuring $5 \times 5 \times 1 \mathrm{~mm}$ mounted on a thermoelectric, two stage cooler [11].

The X-ray tube used is a microfocused, reflection type, with $300 \mathrm{kV}$ maximum tension and a tungsten target [13] that comprises the v|tome|x microtomography system, manufactured by General Electric (GE) [14].

For spectra acquisition the detector was installed within the tomographer in a stable manner, and it was kept aligned with the X-ray window throughout the entire experiment. To align the CdTe detector window and the X-ray tube, the detector system of the tomographer was utilized, which allowed the visualization of the entrance window of the detector and its alignment with the tube through the image shown on the screen of the tomography operation monitor. Figure 1 shows the tube-detector system used.

Figure 1: Tube-detector system used for spectra acquisition. Figure shows the inside of the $v \mid$ tome $\mid x 300$ microtomographer at the Nuclear Instrumentation Laboratory (Laboratorio de Instrumentação Nuclear) in UFRJ. 


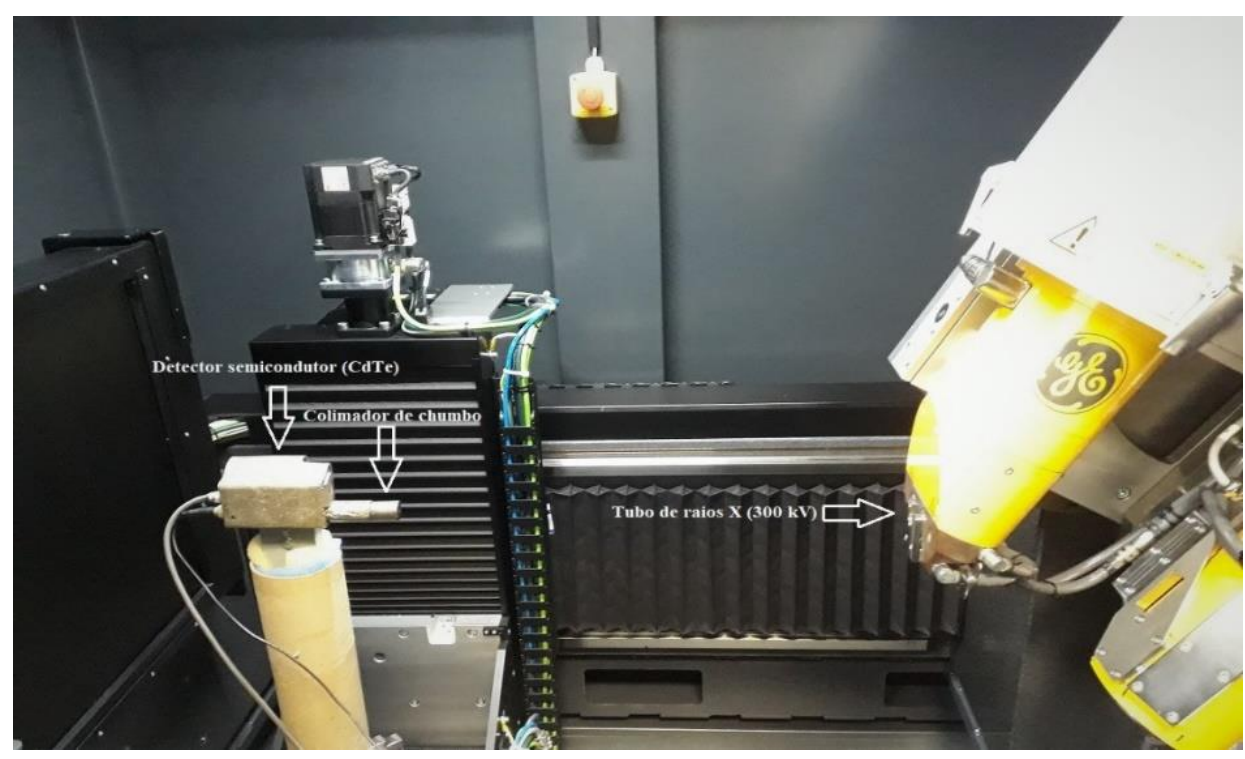

A lead collimator aligned to the tube and the detector window was used to reduce scattered radiation at the entrance of the detector window as a way to lower incident beam intensity in the detector. This intensity reduction is necessary to reduce dead time during spectra acquisition which also avoids imprecisions on the resulting spectrum.

The calibration of the detector was made using a $241 \mathrm{Am}$ source $(95 \mathrm{mCi})$, with a known spectrum. The source emits alpha radiation as well as gamma radiation with energy peaks of 26.3 $\mathrm{keV}$ and $59.6 \mathrm{keV}$ [15], so it was possible to relate each of the multichannel values to a specific energy value.

Aluminum and copper filters used were $0.5 \mathrm{~mm}$ and $0.1 \mathrm{~mm}$ thick, their areas larger than the tube window as to ensure the entire beam would pass through the applied filter. The filtering system was placed alongside the tube window, parallel to the detector and tube windows.

A computer containing the acquisition software ADMCA, from Amptek, was connected to the detection system, which made it possible to analyze and storage each of the acquired spectra. To obtain spectra of satisfactory precision level a 300 second acquisition time was established as well as a dead time limit of $13 \%$. The current inside the X-ray tube throughout the entire experiment was $5 \mu \mathrm{A}$. A threshold was fixated on channel 30 in order to discard any relatively small signal originated from electronic noise. If not discarded, noise signals of this nature would interfere with 
the study, for they are not originated inside the X-ray tube. After spectra acquisition the average energy values ( $E_{\text {average }}$ ) of each X-ray beam was calculated using weighted average (1):

$$
E_{\text {average }}=\frac{\left(\sum E_{i} \times N_{i}\right)}{\sum N_{i}}
$$

Where $E_{i}$ represents the energy at a certain point of the spectrum and $N_{i}$ is the number of counts at the same point. The attenuation value of the beam was also calculated for each filtering system. Attenuation is given by (2):

$$
\text { Attenuation }=1-\frac{I}{I_{0}}
$$

Where $I$ is the intensity of the filtered beam and $I_{0}$ its intensity prior to its passage through the filtering system. Both average energy and attenuation values were calculated using output data generated by the spectra acquisition software.

\section{RESULTS AND DISCUSSIONS}

Results of this work comprise acquired and analyzed energy spectra from a X-ray tube using different tensions and filtering configurations.

\subsection{Unfiltered acquired spectra}

The presence of characteristic energy peaks around $10 \mathrm{keV}$ was observed in all spectra, as well as the appearance of new characteristic energy peaks in the $80 \mathrm{kV}$ spectrum. In Figure 2 it is possible to observe, by inspection of the characteristic energy peak values, that the tube under analysis has a tungsten target [16]. Possible characteristic peak values variations from the ones established in known related literature $[17,18]$ are due to experimental imprecisions.

Figure 2: Energy spectrum at tube potential of $40 \mathrm{kV}$. 


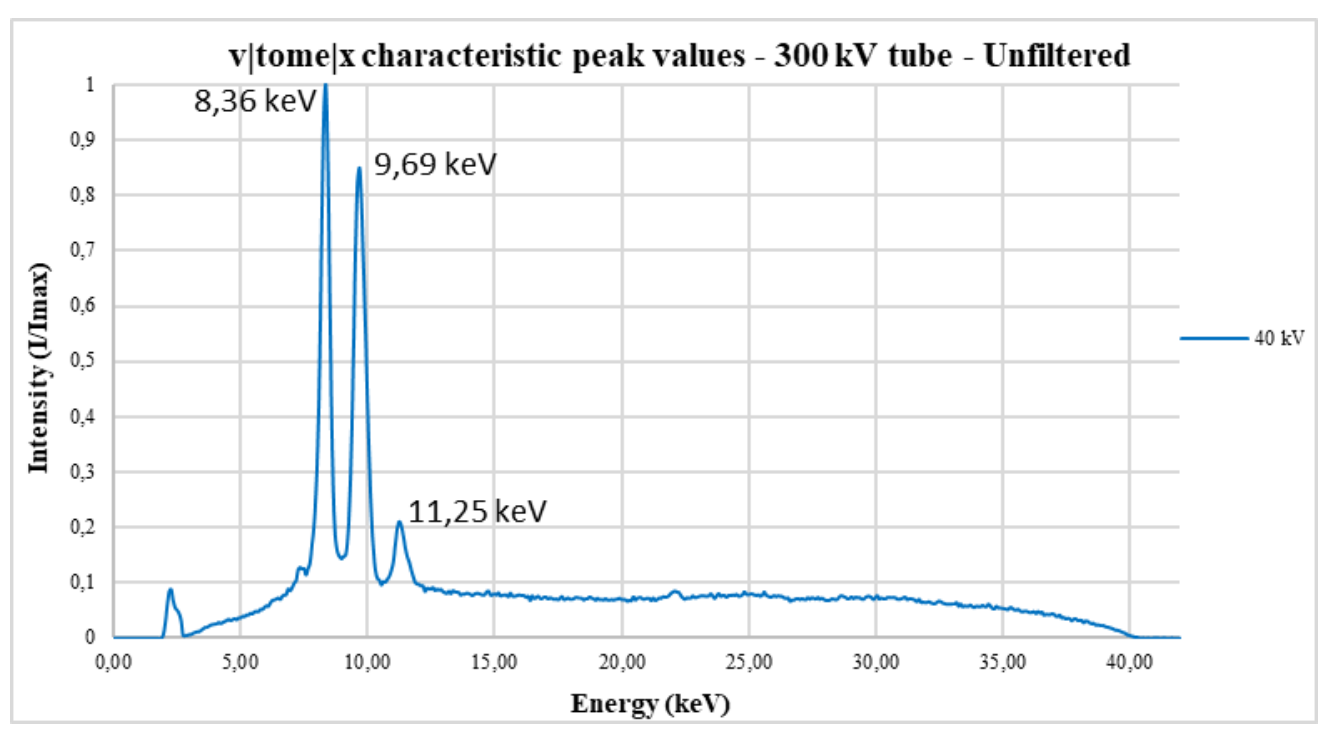

Unfiltered spectra of $20 \mathrm{kV}, 30 \mathrm{kV}, 40 \mathrm{kV}, 50 \mathrm{kV}, 60 \mathrm{kV}, 70 \mathrm{kV}$ and $80 \mathrm{kV}$ were acquired from the v|tome|x m reflection tube. Major potential differences lead to a substantial drop in reliability due to the increase of dead time, making the evaluation of these spectra unfeasible. The acquired unfiltered spectra are presented in Figure 3.

Figure 3: Normalized unfiltered spectra from $v \mid$ tome $\mid x m$ directional tube.

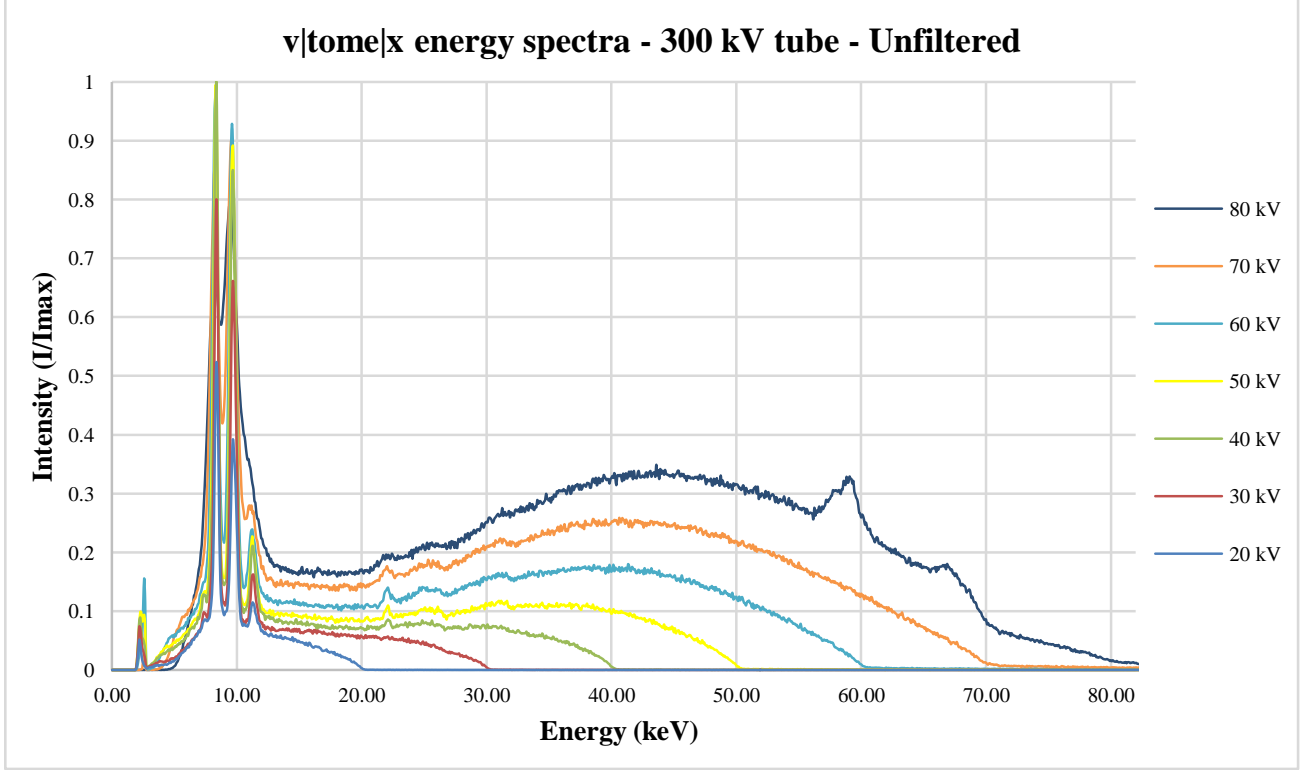




\subsection{Energy spectra with application of filters}

Aluminum and copper were the materials chosen as filtering systems. Both are of great importance to the microCT technique, for they are largely used in microtomography [19].

The acquisition of spectra with voltages above $80 \mathrm{kV}$ were possible using filtering systems, with no reliability loss, which is justified by the attenuation caused by the filter and the non-saturation of the detector under these conditions [12]. Using filters thicker than $0.5 \mathrm{~mm}$ made studying greater potential differences possible, $120 \mathrm{kV}$ being the maximum one, as shown in Figure 4.

Figure 4: $v \mid$ tome $\mid x$ m energy spectra with $0.5 \mathrm{~mm}$ copper filter applied.

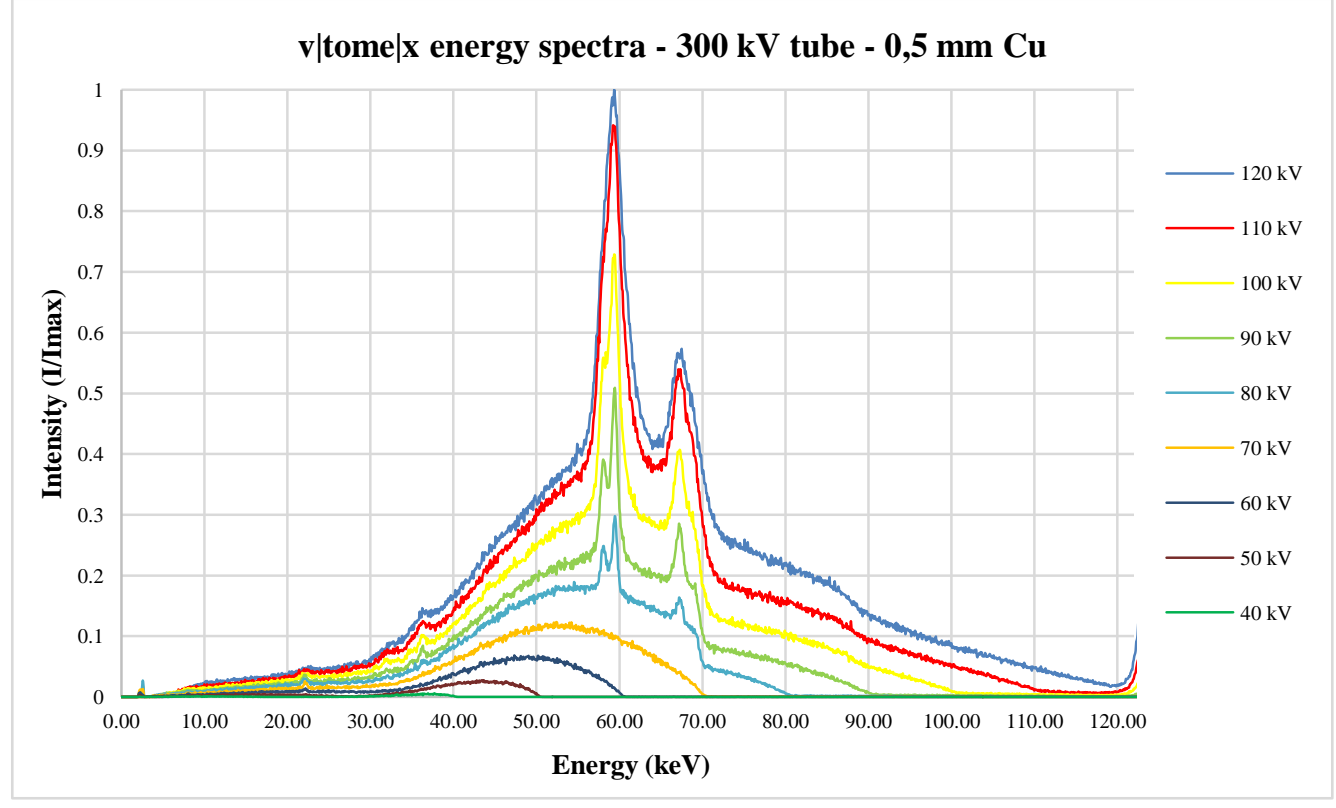

Spectra with maximum energy up to $50 \mathrm{keV}$ were almost entirely attenuated. In these cases, the maximum count was around $2.5 \%$ of the maximum for potential difference of $120 \mathrm{kV}$, as shown in Figure 5 .

Figure 5: Zoom of the spectra region of 40 and $50 \mathrm{kV}$. 


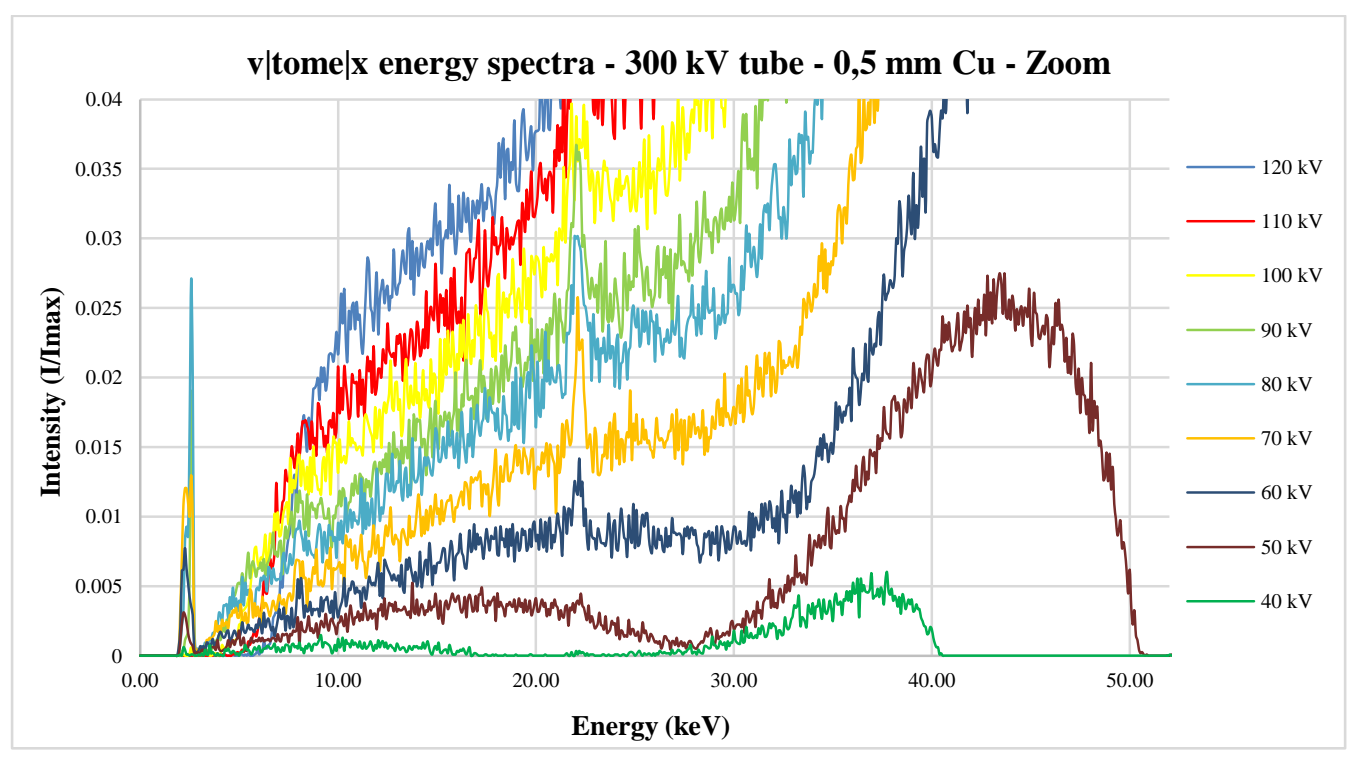

For aluminum only filters the maximum value of applied tension obtained was $90 \mathrm{kV}$, with the purpose of not exceeding dead time limit. The potential limit was slightly higher than the one in the unfiltered case. The difference between limits of voltage reached in copper and aluminum is due to higher attenuation coefficient of copper when compared to aluminum [20]. Figure 6 presents the 40 to $90 \mathrm{kV}$ spectra acquired using a $1.0 \mathrm{~mm}$ aluminum filter.

Figure 6: Energy spectra with 1.0 mm aluminum filter applied.

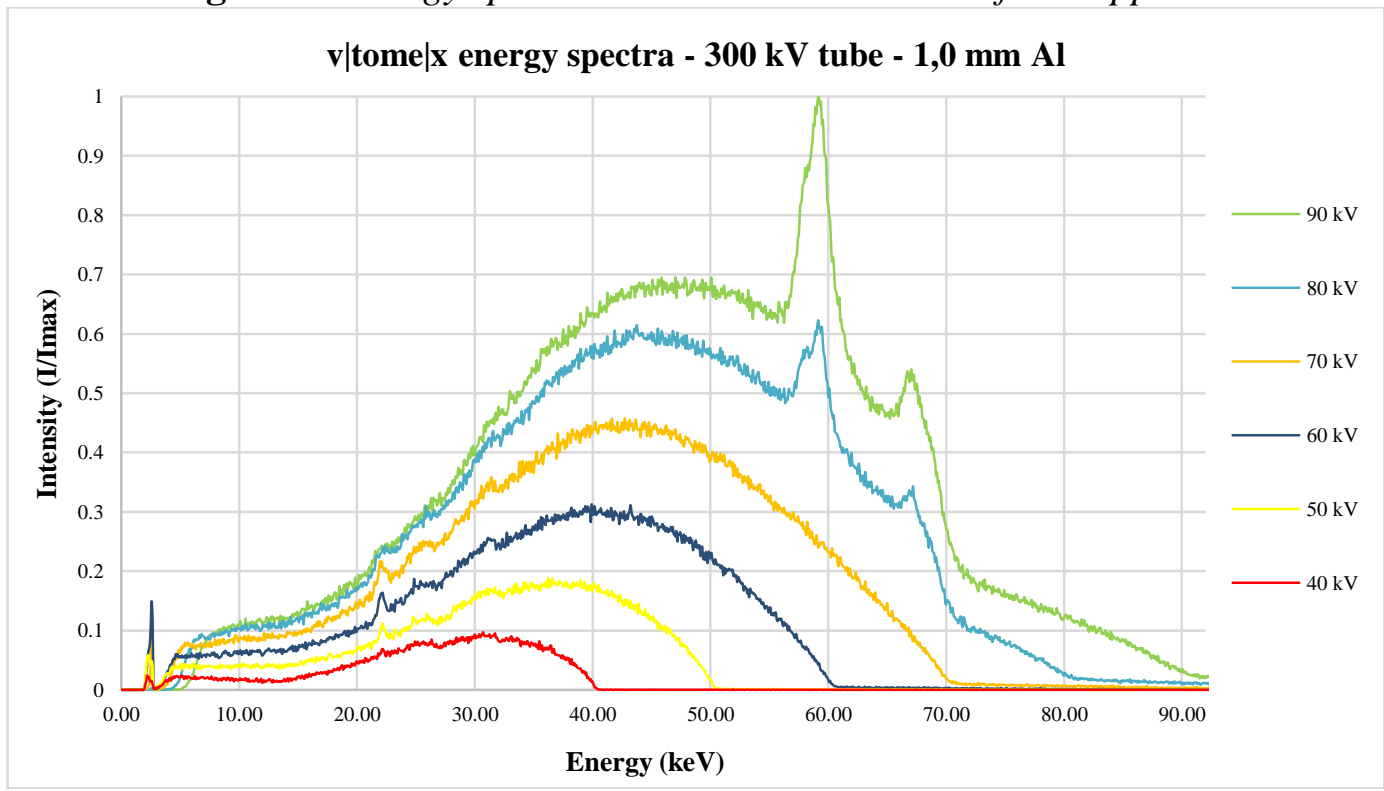




\subsection{Energy spectra at $60 \mathrm{kV}$ tube voltage}

Figure 7 presents the energy spectra for different applied filtering systems at tube voltage of $60 \mathrm{kV}$.

Figure 7: Energy spectra at $60 \mathrm{kV}$ tube potential.

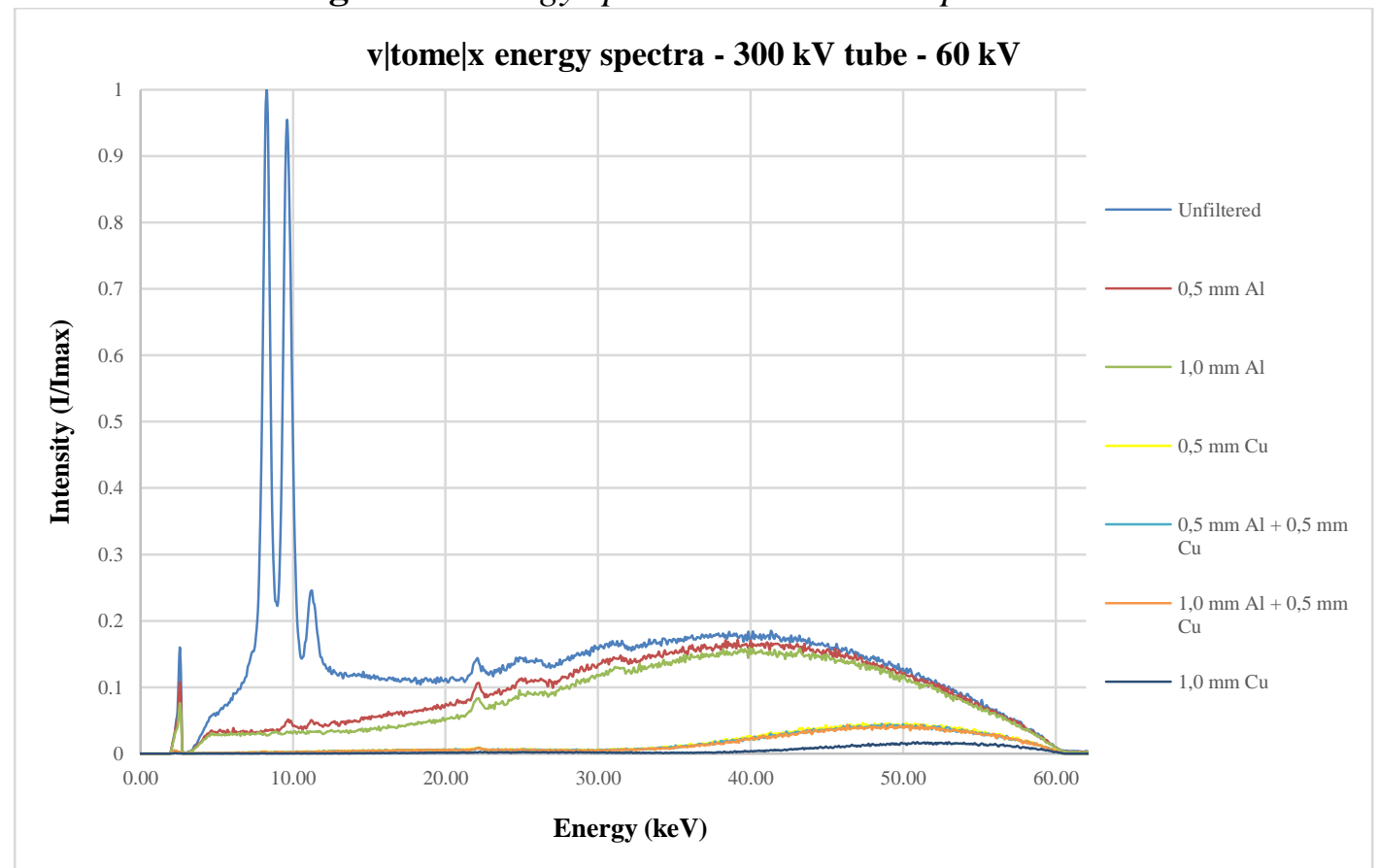

To improve visualization of the spectra in Figure 7, where copper is used in the filtering system, Figure 8 shows the magnification of the area that contains it.

Figure 8: Magnification of region containing copper filter spectra from Figure 7. 


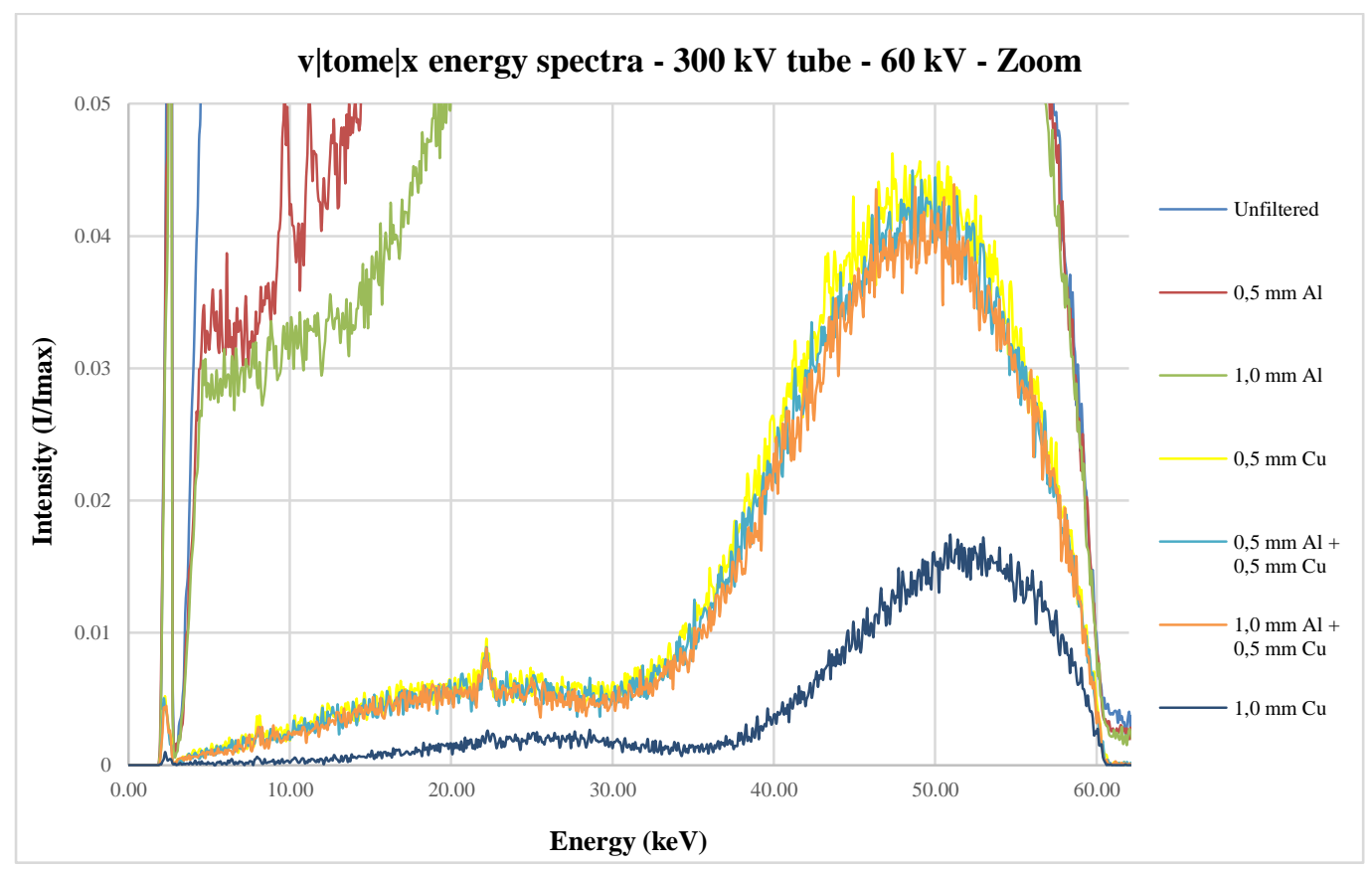

Figure 9 shows attenuation of $60 \mathrm{kV}$ spectra due to application of each evaluated filtering system. Attenuation was calculated for $10 \mathrm{keV}$ intervals so each point in the graph indicates attenuation at that corresponding specific energy interval.

Figure 9: Attenuation by energy interval - $60 \mathrm{kV}$ spectrum.

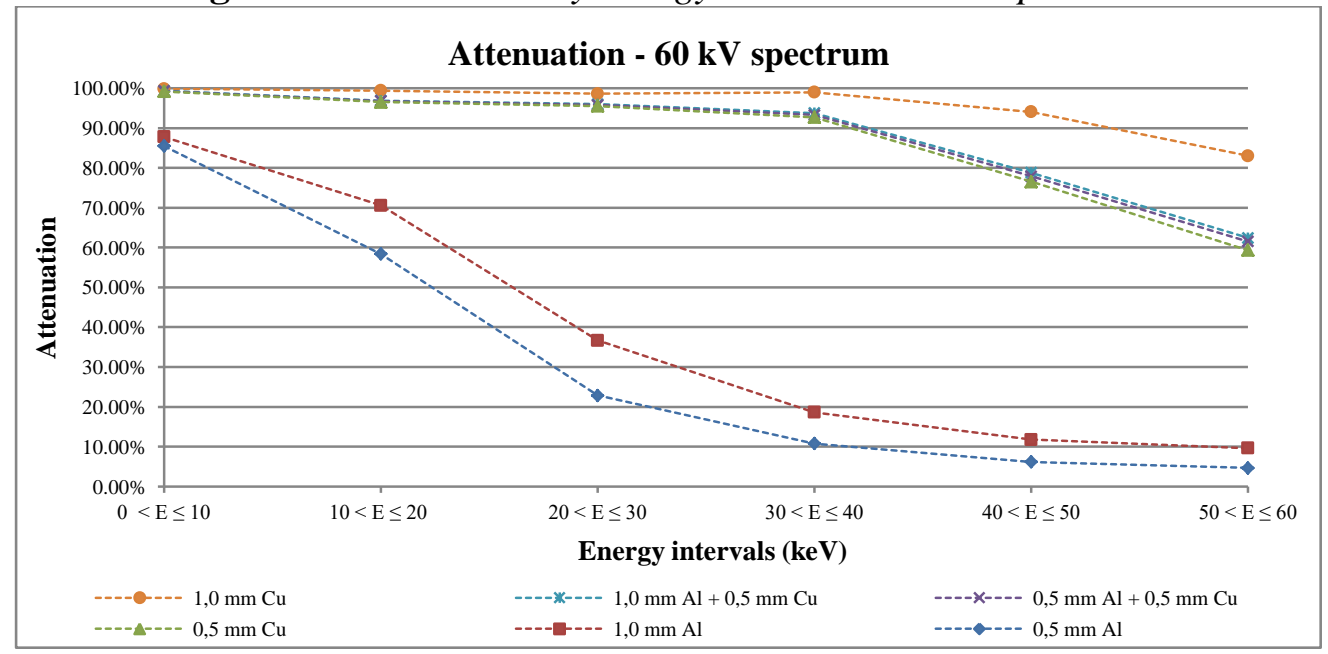




\subsection{Energy spectra at $70 \mathrm{kV}$ tube voltage}

In Figure 10, as in Figure 7, the spectra for each filtering system are presented, however, in this case, at tube voltage of $70 \mathrm{kV}$.

Figure 10: Energy spectra at $70 \mathrm{kV}$ tube potential.

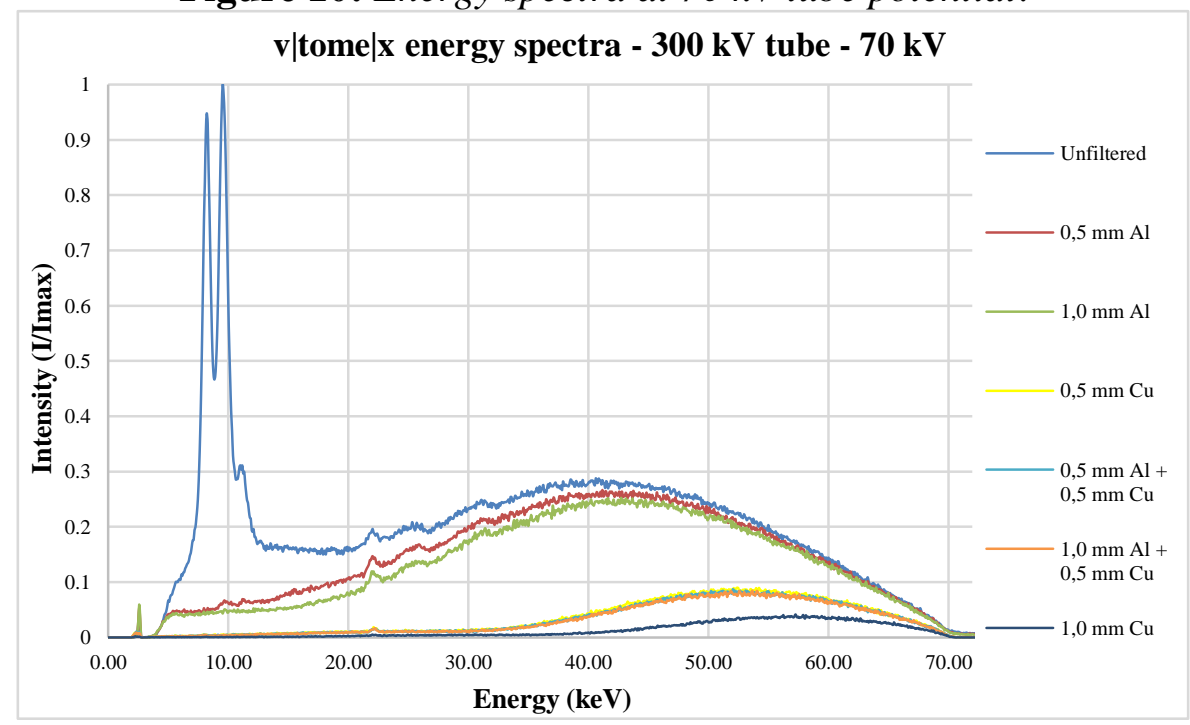

The attenuation suffered by both $60 \mathrm{kV}$ and $70 \mathrm{kV}$ spectra presents a similar behavior, as shown by comparison of Figures 9 and 11 .

Figure 11: Attenuation per energy interval - $70 \mathrm{kV}$ spectrum.

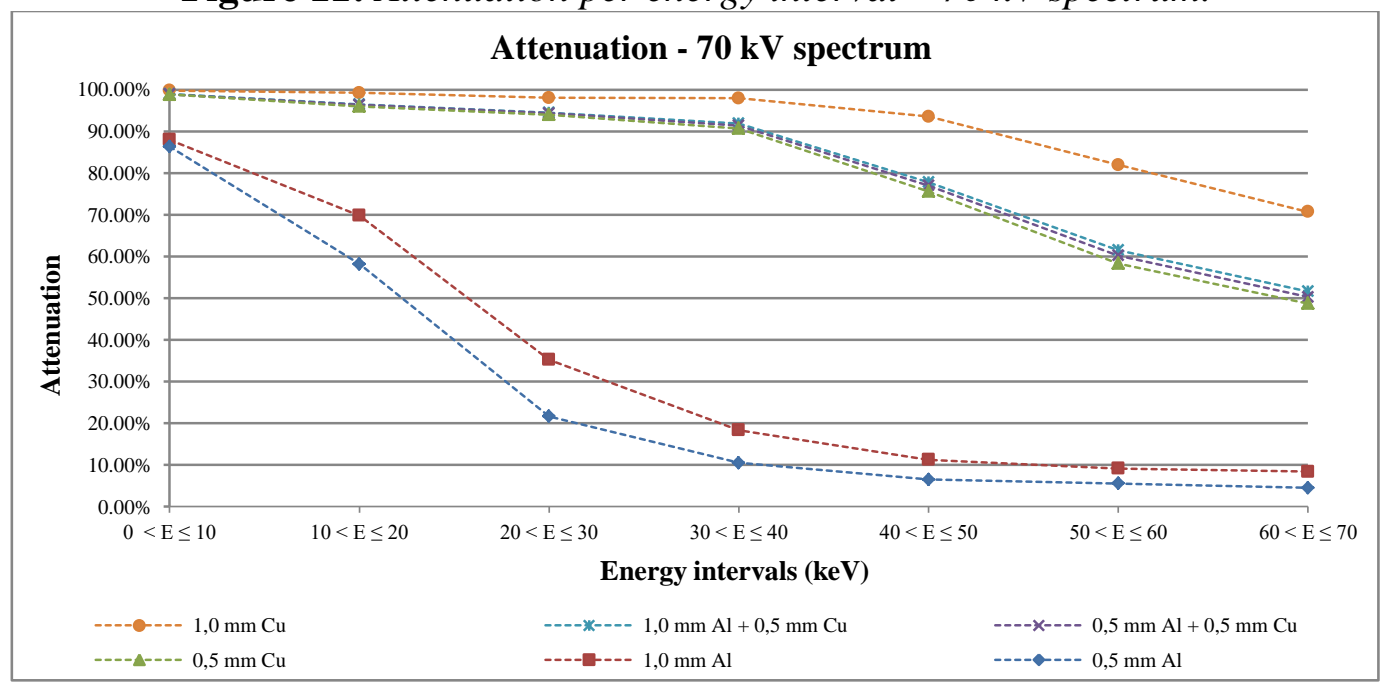




\subsection{Average energies}

Average energy values from each acquired spectra were also calculated. These values are presented in Figure 12 by filtering system and the X-ray tube voltage.

Figure 12: Average beam energy for different voltages and filtering systems.

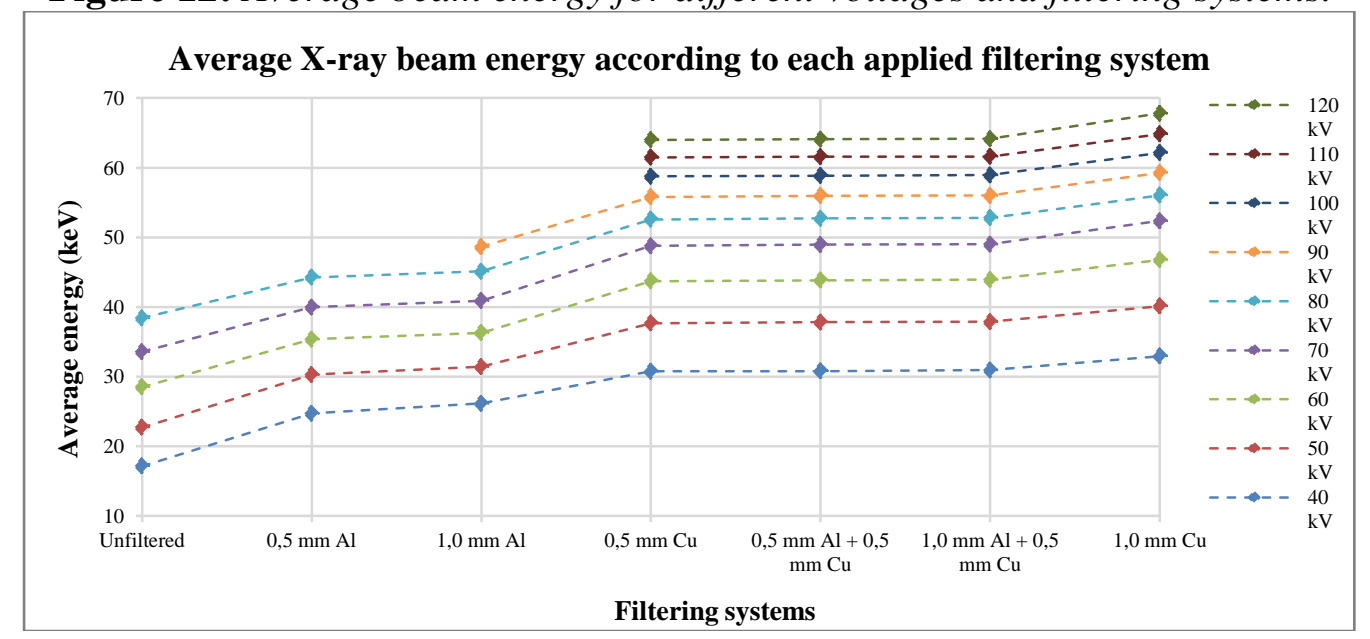

The application of filters caused a similar effect in terms of beam energy average in all analyzed tensions (Figure 12), which can also be observed in Figure 13.

Figure 13: Percentage/absolute increase of the average energy - $60 \mathrm{kV}$ and $70 \mathrm{kV}$ spectra.

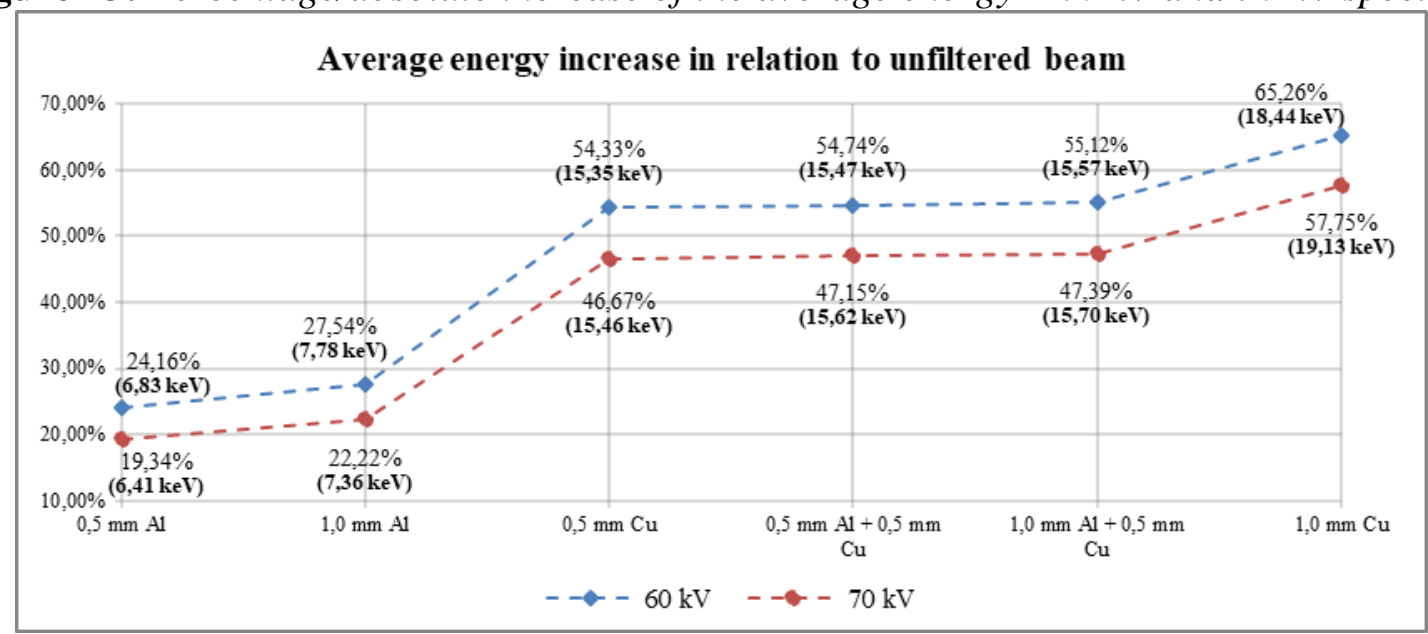

A considerable difference in percentage increase of average energy between $60 \mathrm{kV}$ and $70 \mathrm{kV}$ spectra can be observed (Figure 13). However, in absolute values, the gain in average energy related 
to each filtering system was similar in both analyzed tensions, as seen in the values of $15.35 \mathrm{keV}$ for the $60 \mathrm{kV}$ spectrum and $15.46 \mathrm{keV}$ for the $70 \mathrm{kV}$ spectrum, both using a $0.5 \mathrm{~mm}$ copper filter.

\section{CONCLUSION}

The application of any of the filtering systems resulted in significant increase of average energy when compared to the average energy of the unfiltered beam. This is due to the stronger attenuation of lower energies photons in relation to the attenuation of the more energetic photons of the X-ray beam.

Systems containing copper filter showed greater attenuation throughout the entire spectrum, over $90 \%$ for energy lower than $40 \mathrm{keV}$. Aluminum only filters presented great loss of attenuation capabilities for energies above $20 \mathrm{keV}$, attenuating around $35 \%$ of the $20 \mathrm{keV}$ and $30 \mathrm{keV} \mathrm{X}$-ray beams using the $1.0 \mathrm{~mm}$ aluminum filter. Adding aluminum to copper based filtering systems lead to a slight increase both in attenuation of the system and its average energy gain compared to copper only filtering system.

The use of filters may be interesting when average energy gain of the beam is needed or exposing samples to low energies is undesirable. Aluminum may be used for a smaller, however very effective attenuation of low energies, and copper when higher attenuation is desired, which is also effective for low energies although much more relevant for higher energies.

\section{REFERENCES}

[1] "X ray: Applications of $\quad$ R https://www.infoplease.com/encyclopedia/science/physics/concepts/X-ray/applications-of-X-rays (2020).

[2] M. L. Bouxsein, S. K. Boyd, B. A. Christiansen, R. E. Guldberg, K. J. Jepsen e R. Muller, "Guidelines for assessment of bone microstructure in rodents using micro-computed tomography", Journal of Bone and Mineral Research, vol 25, issue 7 (2010). 
[3] G. De-Deus, F. G. Belladonna, E. J. N. L. Silva, J. R. Marins, E. M. Souza, R. Perez, R. T. Lopes, M. A. Versiani, S. Paciornik e A. A. Neves, "Micro-CT Evaluation of Non-Instrumented Canal Areas with Different Enlargements Performed by NiTi Systems", Brasilian Dental Journal, vol 26 n 6, pp. 624-629 (2015).

[4] Sasai et al.: "The use of micro-computed tomography in the diagnosis of dental and oral disease in rabbits". BMC Veterinary Research 10:209 (2014).

[5] J. M. S. Silva, I. Zanette, P. B. Noël, M. B. Cardoso, M. A. Kimm e F. Pfeiffer, "Threedimensional non-destructive soft-tissue visualization with X-ray staining micro-tomography”, Scientifict Reports, 5:14088 (2015).

[6] C. Vale, M. F. C. Pereira, A. Maurício, B. Vidal, A. Rodrigues, J, Caetano-Lopes, A. Nazarian, J. E. Fonseca, H. Canhão e M. F. Vaz, "Micro-computed tomography assessment of human femoral trabecular bone for two disease groups (fragility fracture and coxarthrosis): Age and gender related effects on the microstructure", Journal of Biomedical Science and Engineering, vol 6(02), pp.175-184 (2013).

[7] Y. Wu, S. Adeeb e M. R. Doschak, "Using Micro-CT Derived Bone Microarchitecture to Analyze Bone Stiffness - A Case Study on Osteoporosis Rat Bone”, Frontiers in Endocrinology, 6:80 (2015).

[8] H. Kim, K. Park, D. Kim, H. Chae, G. Sung e Y. Kim, "In vitro assessments of bone microcomputed tomography in an aged male rat model supplemented with Panax ginseng", Saudi Journal of Biological Sciences, vol 25 n 6, pp. 1135-1139 (2018).

[9] M. A. P. Santos, M. C. F. Fragoso, M. L. Oliveira, R. A. Lima e C. A. Hazin, "The use of CdTe detectors for dental x-ray spectrometry", International Nuclear Atlantic Conference - INAC 2007, Santos, SP, Brazil, September 30 to October 5, 2007.

[10] G. Sato, T. Takahashi, M. Sugiho, M. Kouda, T. Mitani, K. Nakazawa, Y. Okada e S. Watanabe, "Characterization of CdTe/CdZnTe detectors", IEEE Transactions on Nuclear Science, vol 49 n 3, pp. 1258-1263 (2002).

[11] “XR-100CdTe X-Ray \& Gamma Ray Detector”, https://www.amptek.com/products/cdte-Xray-and-gamma-ray-detectors/xr-100cdte-X-ray-and-gamma-ray-detector (2019).

[12] G. F. Knoll, Radiation Detection and Measurement, John Wiley \& Sons, New York \& EUA (2000). 
[13] A. P. Teles, “APLICAÇÃO DA MICROTOMOGRAFIA COMPUTADORIZADA DE RAIOS X POR DUPLA ENERGIA NA CARACTERIZAÇÃO DE MATERIAS POROSOS”. 2016. Tese (Doutorado em Engenharia Nuclear) - UFRJ/ COPPE/ Programa de Engenharia Nuclear.

[14] V|TOME|X M microfocus CT, https://www.industrial.ai/sites/g/files/cozyhq596/files/201906/geit-31319en_vtomexm_brochure_16052019_web.pdf (2019).

[15] Am241 Gamma Spectrum, https://www.gammaspectacular.com/blue/am241-spectrum (2020).

[16] Characteristic emission of lines elements, https://www.chess.cornell.edu/users/calculators/characteristic-emission-lines-elements (2019).

[17] "CdTe Application Note: Characterization of X-ray Tubes", https://www.amptek.com/products/cdte-X-ray-and-gamma-ray-detectors/cdte-application-notecharacterization-of-X-ray-tubes (2020).

[18] D. Zhang, X. Li e B. Liu, "X-ray spectral measurements for tungsten-anode from 20 to 49 kvp on a digital breast tomosynthesis system", Medical Physics, Vol. 39, No. 6, June 2012.

[19] L. Nascimento-Dias, D. F. Oliveira, A. S. Machado, O. M. O. Araújo, R. T. Lopes e M. J. Anjos, "Utilization of nondestructive techniques for analysis of the Martian meteorite NWA 6963 and its implications for astrobiology”, X-RAY SPECTROMETRY, vol 47 n 1, pp.86-91 (2018).

[20] "X-Ray Mass Attenuation Coefficients", https://physics.nist.gov/PhysRefData/XrayMassCoef/tab1.html (2020). 